\title{
LA CADENA CAUSAL DE LA CONFIANZA EN LOS ORGANISMOS ELECTORALES DE AMÉRICA LATINA: SUS DETERMINANTES Y SU IMPACTO SOBRE LA CALIDAD DE LA DEMOCRACIA*
}

\section{The Causal Chain of Trust in Electoral Bodies of Latin America: Its Determinants and Impact on the Quality of Democracy}

\author{
MIKEL BARREDA \\ Universitat Oberta de Catalunya
}

\section{LETICIA M. RUIZ RODRÍGUEZ}

Universidad Complutense de Madrid

\begin{abstract}
RESUMEN
El trabajo se centra en la confianza de los ciudadanos en los organismos electorales latinoamericanos analizando tanto sus causas como su impacto sobre la calidad de la democracia. En primer lugar, se traza un mapa de los variados niveles de confianza en los organismos electorales utilizando datos de opinión pública (LAPOP). En segundo lugar, mediante un análisis booleano se explora el efecto combinado de variables relacionadas con el diseño institucional, el entorno político y la cultura política sobre la confianza en los organismos electorales. En tercer lugar, se estudia el impacto de la confianza en los organismos electorales sobre la calidad de la democracia. Para ello se recurre, entre otras técnicas, a un análisis de regresión lineal.
\end{abstract}

Palabras clave: Organismos electorales, confianza, calidad de la democracia, América Latina.

\begin{abstract}
This paper focuses on the confidence of citizens in Latin American Electoral Bodies by analyzing both its causes and its impact on the quality of democracy. First, a map of the varying levels of trust in Electoral Bodies is drawn using public opinion data (LAPOP). Second, through a boolean analysis it is explored the combined effects of variables related to institutional design, political environment and political culture on trust in Electoral Bodies. Third, it is analyzed the impact of trust in Electoral Bodies on the quality of democracy. For that purpose, a lineal regression analysis has been run, apart from other techniques.
\end{abstract}

Key words: Electoral bodies, trust, quality of democracy, Latin America.

* Este artículo está apoyado por el Proyecto “Vínculos entre electores y partidos: la actividad distrital de los diputados de Chile, Perú y Bolivia" (CSO2011-24344) del que forman parte los autores y que financia el Ministerio de Educación y Ciencia de España. Los autores desean agradecer los comentarios y ayuda de Joan Balcells, Albert Padró-Solanet y Hugo Picado, así como las útiles observaciones de los evaluadores anónimos. 


\section{INTRODUCCIÓN}

Uno de los requisitos elementales de un régimen democrático es la existencia de instituciones que garanticen unos estándares mínimos de libertad, justicia y competitividad en los procesos electorales. Las democracias latinoamericanas no son una excepción. Desde los años ochenta del siglo pasado, los organismos de administración y supervisión electoral de América Latina han desempeñado un papel clave en la mejora de la calidad de las elecciones (Hartlyn, McCoy y Mustillo, 2009; Barrientos, 2011). En este contexto, no resulta extraño el número cada vez mayor de estudios politológicos sobre los organismos electorales (OE) latinoamericanos. No obstante, la investigación sustentada en análisis empíricos aún es bastante limitada. Muchas de las ideas que a menudo se establecen no han sido suficientemente contrastadas y quedan bastantes cuestiones pendientes de analizar. Este artículo, centrado en el estudio de la confianza en los OE de América Latina, pretende ser una contribución en esa dirección.

Tres razones fundamentales justifican el interés de estudiar la confianza ciudadana en los $\mathrm{OE}$. En primer lugar, debido al rol decisivo que cumplen los $\mathrm{OE}$ en los procesos electorales, el examen de la confianza ciudadana en ellos constituye una cuestión de interés académico y social. En la literatura se ha destacado la incidencia de este tipo de confianza sobre la participación electoral y el apoyo general al sistema político (Struwig, Roberts, Vivier, 2011). En un sentido más amplio, se ha considerado la confianza en las instituciones -incluidas las electorales- como un criterio para evaluar la salud de una democracia (Putnam, Pharr y Dalton, 2000; Newton y Norris, 2000).

En segundo lugar, por la confianza ciudadana en los $\mathrm{OE}$ podemos disponer de información sobre el nivel de desempeño de estos organismos. La razón es simple: los ciudadanos expresan mayores o menores grados de confianza en los $\mathrm{OE}$ en función de cómo perciben la eficacia de estos organismos en el cumplimiento de sus funciones. Este argumento es invocado frecuentemente en relación con la confianza en las principales instituciones políticas y hay evidencia empírica que lo sustenta. ${ }^{1}$

Por último, hay una razón de orden "práctico" que motiva la atención a la confianza en los OE: la disponibilidad de indicadores para las dieciocho democracias latinoamericanas. Esta información no solo permitirá realizar una comparación descriptiva del nivel de confianza en los diferentes OE de la región, sino desarrollar un ejercicio más analítico en el que se contemple la confianza en los $\mathrm{OE}$ tanto como variable dependiente como variable independiente. Así, este artículo examina dos cuestiones de interés en esta literatura: los principales factores (institucionales, societales y culturales) que inciden en el desempeño de los $\mathrm{OE}$, y el impacto de los $\mathrm{OE}$ en la calidad de la democracia.

Para analizar comparativamente la confianza en los OE se acude a datos y técnicas cuantitativas (entre otras, análisis de regresión lineal) y cualitativas (análisis booleano).

1 Ver, por ejemplo, Weil (1989), Putnam, Pharr y Dalton (2000). Ello no significa que el rendimiento institucional percibido sea el único factor que influye en la confianza; hay otros factores como el tipo de información accesible para los ciudadanos o los criterios considerados en la evaluación. 
Esta combinación metodológica, original dentro de la literatura sobre $\mathrm{OE}$, permitirá una aproximación más comprehensiva a nuestro objeto de estudio.

De acuerdo con estos propósitos y esta metodología, el artículo se ha dividido en cuatro apartados, además de la presente introducción y las conclusiones. En el siguiente apartado se expone la importancia de los $\mathrm{OE}$ dentro de los sistemas democráticos y se realiza un breve estado del arte. En el tercer apartado se presenta una radiografía de la confianza en los OE latinoamericanos, a partir de datos de encuestas a ciudadanos y diputados. En el cuarto apartado se exploran los factores que afectan a la variación en los niveles de confianza en los OE. Para ello se acude a un análisis cualitativo multicausal (basado en el álgebra booleana). El último apartado examina el impacto de la confianza ciudadana sobre la calidad de las democracias latinoamericanas mediante un análisis estadístico.

\section{LA IMPORTANCIA DE LOS ORGANISMOS ELECTORALES Y LA INVESTIGACIÓN DESARROLLADA}

Los OE son las entidades encargadas de gestionar las elecciones y de resolver los conflictos entre los principales actores participantes. Por lo tanto, desarrollan funciones administrativas y judiciales asociadas a los procesos electorales (Hartlyn, McCoy y Mustillo, 2009; Barrientos, 2010). El diseño institucional para el cumplimiento de estas funciones varía notablemente de unos países a otros: hay diseños que separan ambas funciones en organismos independientes entre sí, mientras que otros las concentran en un mismo organismo (Brenes, 2009; Thompson y Valverde, 2007). Así, en América Latina la justicia electoral se ejerce, en unos casos, por medio de tribunales específicos dentro del Poder Judicial (por ejemplo, en México o Brasil), en otros casos esta función está incluida en la jurisdicción contencioso-administrativa (Colombia y Nicaragua) y en otros está asignada a los mismos órganos responsables de la administración electoral (por ejemplo, en Costa Rica o Uruguay).

Como es sabido, una de las exigencias básicas de una democracia es que se celebren elecciones "libres y justas". Los OE cumplen una función clave al respecto. El desempeño efectivo de estos organismos no garantiza la existencia de elecciones legítimas y de calidad, pero para que las elecciones disfruten de legitimidad y calidad democráticas resulta indispensable la presencia de OE que desarrollen debidamente las tareas de gestión y control electoral encomendadas (Picado, 2006; Barrientos, 2011; Struwig, Roberts y Vivier, 2011). Este rol de los OE suele ser más visible en las democracias emergentes, debido a las frecuentes debilidades de la administración electoral y los elevados niveles de desconfianza existentes entre los actores políticos (Hartlyn, McCoy y Mustillo, 2009).

A pesar del reconocimiento del papel que cumplen los $\mathrm{OE}$, la investigación académica sobre esta temática es bastante limitada. Esto se debe, en buena medida, a que la atención hacia los $\mathrm{OE}$ es resultado del trabajo de observación electoral y asistencia democrática de las últimas décadas (Mozaffar y Schedler, 2002). La mayoría de los profesionales y académicos que han participado en estas actividades ha tendido a considerar los OE como una variable con la que evaluar la integridad de los procesos electorales. Desde luego, 
hay algunas excepciones que -como se verá- han tratado de examinar empíricamente el impacto de los $\mathrm{OE}$ en las elecciones y en los procesos de democratización.

La investigación politológica sobre los $\mathrm{OE}$ se ha desarrollado en tres vertientes principales. La primera de ellas es el análisis comparativo del diseño institucional de los OE. Entre los criterios institucionales sobresale la independencia política de los OE. Se argumenta que la autonomía de los OE respecto de gobiernos y partidos políticos contribuye positivamente a la calidad de los procesos electorales, especialmente si los OE operan en un entorno de alta desconfianza y polarización política (López Pintor, 2000; Hartlyn, McCoy y Mustillo, 2009). Sobre la base de este criterio de independencia, se han construido diversas tipologías de OE que muestran, en el ámbito latinoamericano, el predominio de organismos de tipo mixto o independiente (frente al modelo gubernamental extendido en otras regiones). ${ }^{2}$ Cabe mencionar también los índices de independencia formal de los OE elaborados por otros investigadores (Molina y Hernández, 1998; López Pintor, 2000; Picado, 2006; Wall et al., 2006; Hartlyn, McCoy y Mustillo, 2009; Barrientos, 2010 y 2011).

Una segunda línea de estudio de los OE se ha centrado en analizar sus consecuencias políticas e institucionales. En especial, se han examinado los efectos sobre los procesos electorales y sobre los procesos de democratización. En relación con lo primero, se ha constatado que una gestión eficaz de los OE impacta positivamente sobre la credibilidad electoral y el nivel de participación (Struwig, Roberts y Vivier, 2011) y que la presencia de OE independientes contribuye a mejorar la confianza en las elecciones por parte de los ciudadanos (Molina y Hernández, 1998), de los parlamentarios (Barrientos, 2010) y de los observadores electorales (Hartlyn, McCoy y Mustillo, 2009). Asimismo, estudios como el de Lehoucq y Molina (2002) muestran que la existencia de OE independientes potencian los procesos de democratización (Costa Rica, en este caso), mientras que otros trabajos han destacado que cuando el desempeño de los OE es débil se obstaculiza el proceso de democratización (Hartlyn, 1998).

Finalmente, una tercera línea de investigaciones ha examinado los OE como variable dependiente. Al igual que en otras instituciones democráticas, la creación y el desempeño de los $\mathrm{OE}$ viene determinado por un conjunto diverso de factores históricos, institucionales, culturales y socioeconómicos (Mozaffar y Schedler, 2002). En especial, se han resaltado dos tipos de factores explicativos. Por un lado, se ha puesto énfasis en el propio diseño institucional y organizativo de los $\mathrm{OE}$, dirigiendo la atención a cuestiones como el nivel de autonomía política, burocratización, centralización o especialización (Mozaffar y Schedler, 2002; Thompson y Valverde, 2007). Por otro lado, se ha destacado la relevancia del contexto político pos y preelectoral y, particularmente, del nivel de competencia política y de litigiosidad electoral (Brenes, 2009).

2 Barrientos $(2010 ; 2011)$ diferencia tres modelos de OE: 1) independiente: las elecciones son organizadas y gestionadas por un OE institucionalmente independiente del poder ejecutivo; 2) gubernamental: las elecciones son organizadas y gestionadas por la rama del poder ejecutivo por un ministerio o secretaría y/o mediante autoridades locales; 3) mixto: suele estar formado por una estructura dual de un OE independiente, generalmente con funciones de supervisión y vigilancia de los procesos, y un OE gubernamental con tareas de gestión y organización electoral. 


\section{UN ANÁLISIS COMPARADO DE LA CONFIANZA EN LOS ORGANISMOS ELECTORALES LATINOAMERICANOS}

En este apartado se examinan de forma comparada los niveles de confianza en los $\mathrm{OE}$ de las dieciocho democracias de la región y su variación a lo largo del tiempo. Consideramos la confianza ciudadana en los OE como una forma de apoyo "específico" más que una forma de apoyo "difuso", de acuerdo con la terminología de Easton. ${ }^{3}$ Para la comparación se ha elegido una de las fuentes más completas sobre confianza en los OE latinoamericanos: la encuesta de opinión que realiza LAPOP (Universidad de Vanderbilt). Esta encuesta incluye desde 2004 una pregunta sobre el nivel de confianza en el Tribunal Supremo de Elecciones de cada país.

En el último de los Barómetros (2010), el promedio de los países latinoamericanos es 4,2 en una escala de 1 ("nada de confianza") a 7 ("mucha"). Se trata de un nivel de confianza aceptable (equivalente a un 6, en una escala de 0 a 10), sobre todo en un escenario donde las instituciones políticas y sociales reciben valoraciones modestas. En efecto, de catorce instituciones, los OE se sitúan por encima del promedio regional (4) y en cuarta posición por orden de mayor a menor nivel de confianza. La Iglesia, el Ejército y los medios de comunicación son las instituciones que aventajan a los OE por un margen estrecho. Asimismo, el promedio regional de confianza en los OE apenas ha variado desde el primer Barómetro de LAPOP (4,1 en 2004, 4 en 2006 y 3,9 en 2008).

Este panorama cambia notablemente en la comparación por países (ver Gráfico 1). Argentina, Paraguay y Nicaragua son los países con niveles más bajos de confianza en los OE; en los tres casos el nivel de confianza está por debajo de 3,5 (el punto medio de la escala). Uruguay se sitúa justo en el polo opuesto: presenta un nivel de confianza en los $\mathrm{OE}(5,3)$ muy por encima de la media regional. Los catorce países restantes se ubican entre estas dos posiciones extremas, aunque con diferencias destacadas entre ellos. Mientras que Ecuador, Perú, Venezuela, Colombia, Brasil y Guatemala se hallan por debajo de la media latinoamericana, los demás países se sitúan por encima, especialmente Chile, Panamá y Costa Rica. En definitiva, el análisis comparativo por países evidencia diferencias significativas. ${ }^{4}$

Además de las percepciones de la ciudadanía, resulta de interés atender a las percepciones de otro actor directamente afectado por la gestión de los OE: los representantes políticos. La base de datos PELA (Universidad de Salamanca) facilita información al respecto. Si comparamos las percepciones de los diputados y las de los ciudadanos durante el mismo

3 El apoyo específico es aquel que reciben las autoridades como resultado de su desempeño, mientras que el apoyo difuso es un apoyo más general y perdurable que disfrutan las autoridades como instituciones de un sistema político (Easton, 1975). La confianza en las instituciones corresponde, según Easton, a un tipo de apoyo difuso. Sin embargo, diversos estudios han evidenciado que los indicadores de confianza institucional utilizados habitualmente en las encuestas capturan más bien la confianza como apoyo específico (Weil, 1989; Aydin y Cenker, 2012).

4 Hemos aplicado un test de diferencias de medias y, a partir de diferencias de 0,2, estas resultan significativas. 


\section{Gráfico 1. Confianza ciudadana en OE en América Latina (2010*}

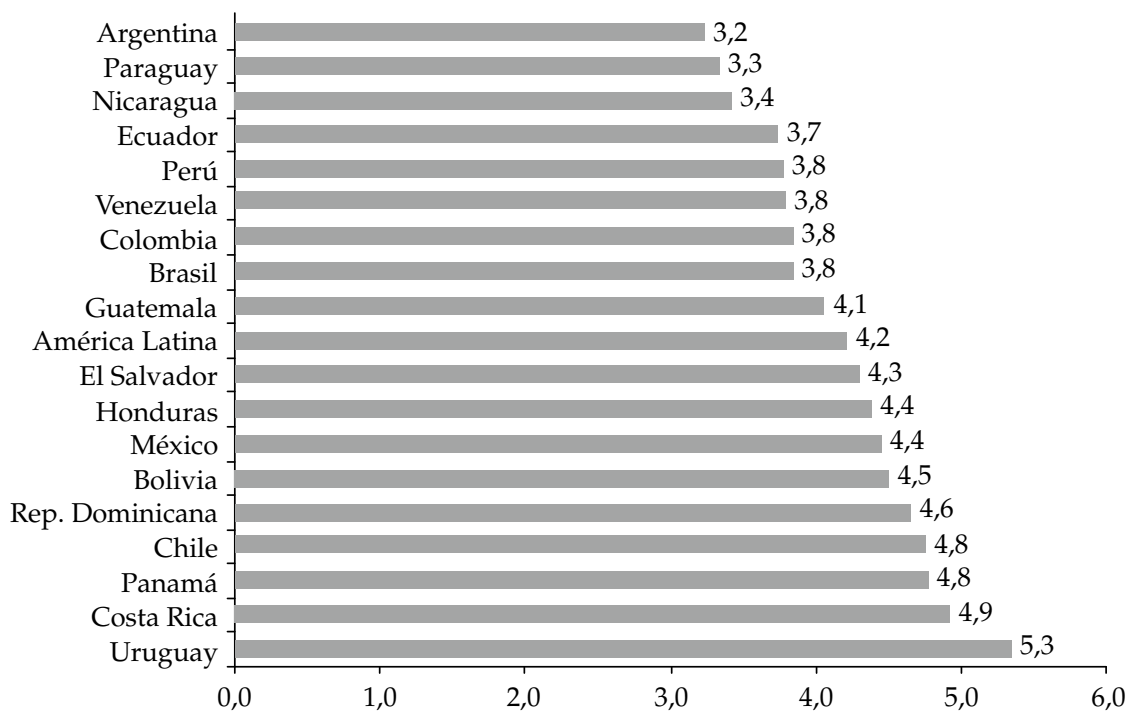

${ }^{*}$ ) Promedio del nivel de confianza en una escala de 1 (nada) a 7 (mucha).

Fuente: elaboración propia a partir de LAPOP.

periodo (2005-08) se obtiene un panorama muy similar. ${ }^{5}$ El nivel de confianza en los OE que expresan, en término medio, los ciudadanos latinoamericanos es prácticamente el mismo que el de sus diputados (4 y 4,1, respectivamente). A su vez, el coeficiente de correlación de Pearson entre los dos indicadores es 0,6 (significativo al 0,05), lo que muestra que hay una destacada asociación positiva entre el nivel de confianza en los OE de los ciudadanos y el de los diputados (ver Gráfico 2). Por tanto, puede hablarse de una sintonía general entre las valoraciones que realizan los ciudadanos latinoamericanos de los $\mathrm{OE}$ y las que hacen los legisladores.

El Gráfico 2 muestra, con más detalle, la sintonía entre las percepciones de los ciudadanos y los diputados en relación con los OE. Así, países como Uruguay, Costa Rica y Chile que despuntan por sus mayores niveles de confianza ciudadana también sobresalen en los niveles de confianza de los parlamentarios, mientras que Paraguay, Honduras y Nicaragua se encuentran en la situación inversa. La posición de Venezuela coincide justo con la media regional. Hay algunos países que se apartan de este patrón. El caso más destacado es Argentina, donde los ciudadanos expresan una baja confianza en los $\mathrm{OE}$ (valor de 3,3), muy al contrario de lo que hacen sus diputados (5,7; la segunda valoración más satisfactoria de toda la región).

Para facilitar la comparación se ha tomado un periodo semejante en ambos casos (2006-2008 en LAPOP y 20052008 en PELA) y se han transformado los valores promedios de cada país de la encuesta de PELA (porcentajes de respuestas de "mucha" y "bastante") según la escala utilizada en LAPOP. En los dos casos los valores de cada país oscilan entre 1 y 7. 
Gráfico 2. Confianza ciudadana y de élites parlamentarias en OE en América Latina $(2005-2008)^{*}$

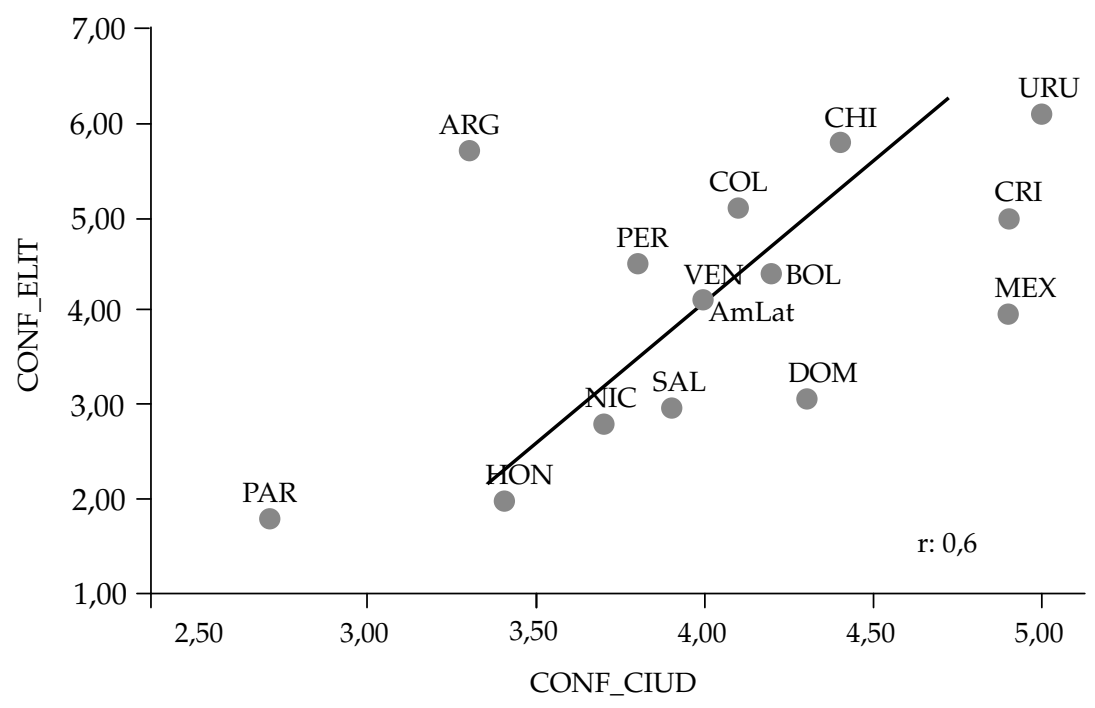

$\left.{ }^{*}\right)$ Promedio del nivel de confianza en una escala de 1 (nada) a 7 (mucha). N: 14 . El coeficiente de correlación de Pearson es significativo al 0,05.

Fuente: elaboración propia a partir de LAPOP (promedio 2006-08), y de Brenes (2009) para datos de PELA (promedio 2005-08).

Como se mencionaba antes, el promedio regional de confianza ciudadana en los OE prácticamente no ha variado desde 2004 a 2010. No obstante, en algunos países el nivel de confianza ha experimentado una mayor fluctuación. Es el caso de la República Dominicana, Honduras, Panamá, Paraguay y, en menor medida, México (ver Gráfico 3). En los cuatro primeros se observa una tendencia a aumentar el nivel de confianza en los últimos años de la serie, justo lo contrario de lo que sucede en México. Estas y otras oscilaciones en el nivel de confianza en los OE se explican, en buena medida, por cambios y ajustes en el diseño institucional de los $\mathrm{OE}$ y por circunstancias específicas de cada país. Un caso muy ilustrativo es México. Las elecciones presidenciales de 2006 tuvieron lugar en un ambiente de notable crispación y se saldaron con la victoria del candidato en el gobierno, Felipe Calderón, por un margen muy estrecho respecto de Andrés López Obrador, lo que llevó a este a impugnar los resultados (Beltrán, 2009). La resolución del Tribunal Electoral del Poder Judicial de la Federación (TEPJF) de validar los resultados originales despertó fuertes protestas entre los partidos de López Obrador. En este contexto, no es casual el descenso del nivel de confianza ciudadana en el TEPJF: de un valor de 5 en 2006 se llega a 4,4 en 2010 (LAPOP). El decremento de la confianza es aún mayor entre los diputados: en 2003 el 85,5\% de los diputados expresaban "mucha" o "bastante" confianza en el TEPJF; en 2006 esta cifra baja hasta el 56,8\% (Brenes, 2009). 


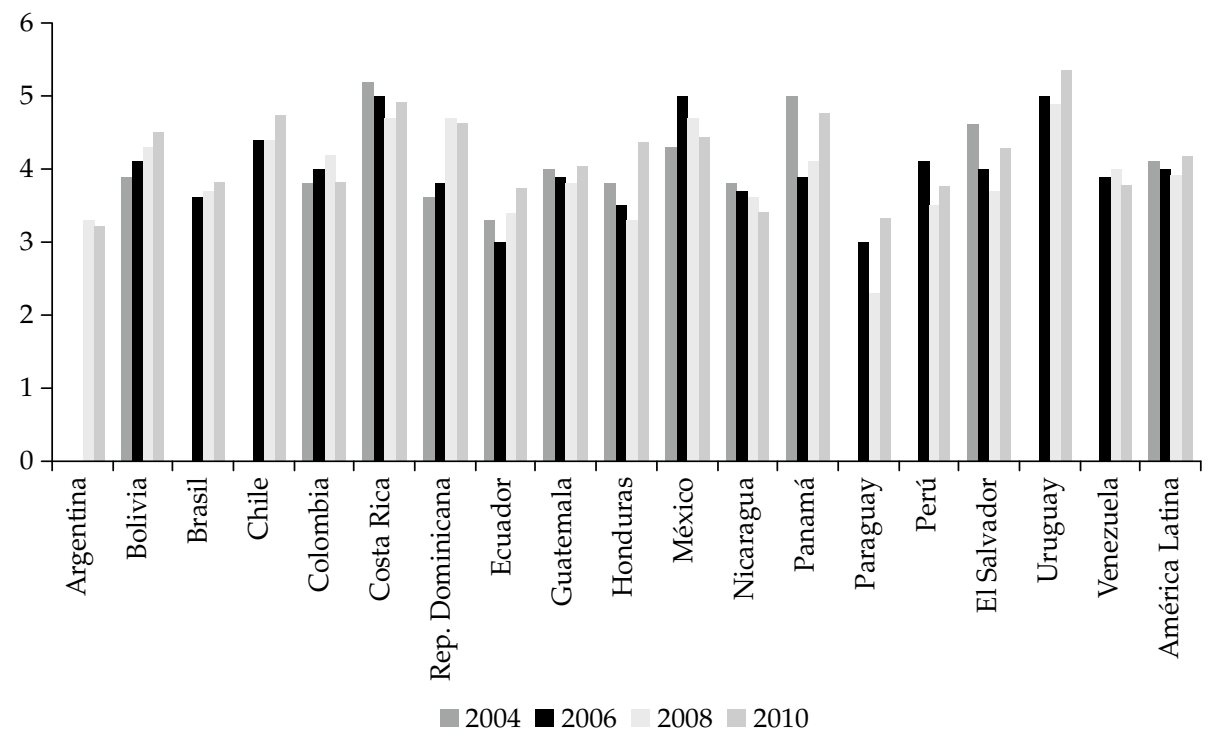

${ }^{*}$ ) Promedio del nivel de confianza en una escala de 1 (nada) a 7 (mucha).

Fuente: elaboración propia a partir de LAPOP.

\section{UNA EXPLORACIÓN DE LOS DETERMINANTES DE LA CONFIANZA EN LOS ORGANISMOS ELECTORALES}

En el apartado anterior se ponía de manifiesto la notable variación que existe respecto del nivel de confianza ciudadana en los distintos $\mathrm{OE}$ latinoamericanos. Los factores que pueden explicar estas diferencias entre casos son diversos. En este apartado se valora el impacto de tres bloques de variables sobre la confianza en los OE: el diseño institucional de los $\mathrm{OE}$, el contexto político-electoral en que operan y la cultura política del país.

La literatura especializada ha apuntado el efecto de estas variables pero no se ha analizado de forma sistemática y con respaldo empírico. Además, los estudios que han examinado estos factores suelen considerar sus efectos de forma aislada. En este trabajo se ha optado por un análisis que combina tres grupos de factores explicativos, en la línea del estudio de Barrientos (2010) sobre la confianza de las élites parlamentarias en los OE y del trabajo de Hartlyn, McCoy y Bustillo (2009) sobre la calidad de las elecciones de la región. Para ello recurrimos a un método comparativo de tipo cualitativo en boga en las ciencias sociales: el análisis booleano. Este tipo de procedimiento permite comparar un número reducido de casos con la intención de identificar pautas causales de ciertos fenómenos (Ragin, 1987).

Con el fin de examinar el impacto de los tres tipos de variables se construirá una tabla de verdad que recogerá las posibles combinaciones de variables independientes 
que dan lugar a mayores o menores niveles de confianza en los OE. Este tipo de tablas facilitan el análisis de los casos, así como un grado de generalización explicativa gracias a la interpretación comprehensiva de los casos y de las variables implicadas en ellos (Ragin, 1987).

A continuación, se presenta la selección de variables independientes elegidas para la explicación de los distintos niveles de confianza en los OE y los valores que estas adquieren en los países latinoamericanos. Los datos han sido obtenidos mediante análisis de las Constituciones y de las leyes electorales, bases de datos sobre elecciones y otras fuentes secundarias, así como de consultas a expertos. ${ }^{6}$ Después de esto se plantea la hipótesis que conjuga el impacto de las variables explicativas elegidas, así como la tabla de verdad construida. Finalmente se señalan los principales hallazgos del análisis booleano.

\section{1. Análisis booleano: diseño institucional de los $\mathrm{OE}$, contexto político-electoral y cultura política como variables explicativas}

\section{El diseño institucional de los $\mathrm{OE}$}

Como se ha comentado, en América Latina ha habido una tendencia a introducir organismos especializados e independientes dedicados a gestionar las elecciones y resolver los posibles conflictos electorales. A pesar de ello, existen notables diferencias en la estructura interna y grado de concentración de funciones de los $\mathrm{OE}$ de la región (Thompson y Valverde, 2007), en el grado de independencia de los mismos respecto de otros poderes y actores, así como en la autonomía de su gestión, por citar algunos factores relevantes de variación entre los OE (Mozzafar y Schedler, 2002). La Tabla 1 muestra la distribución de funciones en materia de administración electoral en los $\mathrm{OE}$ incluidos en el análisis.

El análisis centra la atención en la especialización y en la autonomía de los OE por ser dos variables destacadas del diseño institucional con potencial impacto sobre la confianza en los OE. A juzgar por el rumbo de las reformas en los OE, sería esperable que un diseño institucional que favorezca la especialización y autonomía de los OE en relación con otros actores y poderes contribuyese a aumentar la confianza ciudadana en los OE. Para comprobarlo se medirá la especialización de los $\mathrm{OE}$, distinguiendo entre los que concentran funciones contenciosas y administrativas respecto de los que tienen órganos diferentes para ambas funciones. Además, se analizará la autonomía con dos mediciones: la autonomía en relación con otros poderes, en concreto, la posibilidad de recurrir a las decisiones de los OE ante otras instancias (del Poder Judicial o ante el

6 El punto de partida en la búsqueda de información ha sido la base de datos electorales de CAPEL (http:// www.iidh.ed.cr/capel/), completada con la revisión de otros documentos y consulta a expertos. 
Tabla 1. Organismos electorales: función administrativa y función contenciosa

\begin{tabular}{llc}
\hline & \multicolumn{1}{c}{ Función administrativa } & Función contenciosa \\
\hline Argentina & Dirección Nacional Electoral & Cámara Nacional Electoral (parte del P. Judicial) \\
\hline Bolivia & \multicolumn{2}{c}{ Órgano Electoral Plurinacional-Tribunal Supremo Electoral } \\
\hline Brasil & Tribunal Superior Electoral de Brasil (parte del P. Judicial) \\
\hline Chile & Servicio Electoral & Tribunal Calificador de Elecciones \\
\hline Colombia & & Consejo Nacional Electoral de Colombia \\
\hline Costa Rica & & Tribunal Supremo de Elecciones \\
\hline Ecuador & Consejo Nacional Electoral & Tribunal Contencioso Electoral \\
\hline El Salvador & & Tribunal Supremo Electoral \\
\hline Guatemala & Tribunal Supremo Electoral de Guatemala \\
\hline Honduras & \multicolumn{2}{c}{ Tribunal Supremo Electoral } \\
\hline México & Instituto Federal Electoral & Tribunal Electoral del Poder Judicial de la \\
\hline Nicaragua & & Federación \\
\hline Panamá & Tribunal Electoral & Tribunal Electoral \\
\hline Paraguay & Tribunal Superior de Justicia Electoral (parte del P. Judicial) \\
\hline Perú & Organización Nacional de & Jurado Nacional de Elecciones \\
\hline R. Dominicana & Junta Central Electoral & Suprema Corte de Justicia \\
\hline Uruguay & & Corte Electoral \\
\hline Venezuela & Consejo Nacional Electoral & Sala Electoral del Tribunal Supremo de Justicia \\
\hline
\end{tabular}

Fuente: elaboración propia. Agradecemos a Hugo Picado (TSE) de Costa Rica su ayuda en la revisión de la misma.

Tribunal Constitucional); y la autonomía en relación con otros actores, en particular los vínculos de los $\mathrm{OE}$ con los partidos políticos. ${ }^{7}$

Especialización de los OE: Como se ha señalado anteriormente, hay OE que cumplen funciones administrativas y contenciosas simultáneamente, mientras que otros están

7 Se han sopesado otros posibles indicadores. Por ejemplo, para medir el grado de autonomía respecto del Parlamento se podría utilizar la aprobación del presupuesto de forma autónoma por parte del OE o dependiente del Parlamento. Sin embargo, analizando el valor que adquiere esta variable en los diferentes países, Barrientos (2010) muestra que no hay ningún caso donde no dependa del Parlamento en algún grado. De ahí que no se haya incluido. Otro posible indicador de la independencia respecto de los diferentes poderes sería la estabilidad en el cargo. Sin embargo, la variación de situaciones (por ejemplo, inamovilidad de Argentina, breves períodos de Brasil o más largos de Panamá) hace difícil clasificar esta variable. Sobre estas cuestiones ver Orozco (s.f.). 
conformados por varios organismos con tareas especializadas (Thompson y Valverde, 2007). Mozzafar y Schedler (2002) señalan que la especialización de los OE no tiene efectos claros y unidireccionales sobre el buen funcionamiento de la administración electoral. En nuestra codificación los casos con valor 0 indican que no hay especialización de funciones entre varios $\mathrm{OE}$ (uno solo concentra todas las competencias) y los de valor 1 indican que existe especialización de funciones entre varios $\mathrm{OE}$.

Autonomía en las actuaciones de los OE. La autonomía de los OE se puede medir por la ausencia de intromisiones en el ejercicio de sus funciones y decisiones. Un OE cuyas decisiones son revisadas por otras instancias es esperable que provoque desconfianza en los ciudadanos. Esta variable de la autonomía de los $\mathrm{OE}$ está a caballo entre los criterios de delegación y de regulación de Mozzafar y Schedler (2002). En este trabajo la medimos atendiendo a la recurribilidad de las decisiones del $\mathrm{OE}$ ante otra instancia del poder judicial o ante el Tribunal Constitucional. Esta posibilidad constituye una injerencia de otro órgano en su esfera de decisión que limita la autonomía del mismo. Los casos con valor 0 indican que no se puede recurrir a ninguna decisión en materia electoral y los de valor 1 indican que son recurribles, al menos algunas decisiones en materia electoral, ante el Poder Judicial o ante el Tribunal Constitucional.

Vinculos de los magistrados con los partidos políticos. La autonomía de los OE también se puede medir en relación con los actores del sistema político, en este caso respecto de los partidos políticos. El impacto de los partidos políticos sobre el proceso electoral y sobre la calidad de las elecciones se ha evidenciado repetidamente (Hartlyn, McCoy y Mustillo, 2009). En lo que respecta a la confianza en los OE, la relación de los integrantes de los partidos con los OE cobra relevancia, ya que puede despertar suspicacias respecto de la imparcialidad de sus actuaciones y afectar su nivel de confianza. Cabría pensar que cuanto menor sea la dependencia en relación con los partidos mayor será la confianza en los OE. En la codificación se ha asignado un valor de 0 para los países que establecen que los magistrados no pueden tener vínculos con los partidos y un valor de 1 cuando formalmente se permiten tales vínculos. Cuando el OE está formado por más de un organismo se refiere a los vínculos de los partidos con, al menos, uno de ellos.

La Tabla 2 presenta los valores de los OE de todos los países en relación con las tres variables de diseño institucional examinadas.

\section{El contexto político-electoral}

La confianza en los OE se puede ver afectada también por el contexto político-electoral. Este aspecto ha sido recientemente reivindicado en trabajos sobre OE y calidad de las elecciones (Hartlyn McCoy y Mustillo, 2009; Brenes, 2011). Para analizar el impacto de esta variable se han seleccionado tres indicadores relativos al grado de conflictividad y estabilidad del ambiente político y electoral. Estos factores pueden condicionar las percepciones de los ciudadanos respecto de las instituciones, en general, y sobre los $\mathrm{OE}$, en particular. Se trata de la competitividad electoral, la polarización parlamentaria ponderada y la volatilidad electoral. 
Tabla 2. Variables de diseño institucional de los $\mathrm{OE}$

\begin{tabular}{lccc}
\hline & $\begin{array}{c}\text { Especialización de } \\
\text { funciones del OE }\end{array}$ & $\begin{array}{c}\text { Decisiones del } \\
\text { OE recurribles }\end{array}$ & $\begin{array}{c}\text { Vínculo del OE con } \\
\text { partidos políticos }\end{array}$ \\
\hline Argentina & 1 & 1 & 0 \\
Bolivia & 1 & 1 & 0 \\
Brasil & 1 & 1 & 0 \\
Chile & 1 & 0 & 0 \\
Colombia & 1 & 1 & 1 \\
Costa Rica & 0 & 0 & 0 \\
Ecuador & 1 & 0 & 0 \\
El Salvador & 0 & 1 & 1 \\
Guatemala & 0 & 1 & 0 \\
Honduras & 0 & 1 & 0 \\
México & 1 & 1 & 0 \\
Nicaragua & 0 & 1 & 1 \\
Panamá & 1 & 0 & 0 \\
Paraguay & 0 & 1 & 0 \\
Perú & 1 & 1 & 0 \\
R. Dominicana & 1 & 1 & 0 \\
Uruguay & 0 & 0 & 1 \\
Venezuela & 1 & 1 & 1 \\
\hline
\end{tabular}

Fuente: elaboración propia.

Competitividad electoral. El margen de victoria de un partido político afecta a la dinámica política y electoral. Los resultados electorales estrechos propician una alta litigiosidad electoral (Araujo et al., 2008; Brenes, 2011). Todo ello suele devenir en valoraciones opuestas de la actuación de los OE en la resolución del conflicto (Barrientos, 2008; Sonnleitner, 2007) que contribuyen a debilitar la confianza en los OE. Se mide la competitividad electoral como la diferencia de votos entre el primer y el segundo partido más votados en las últimas elecciones parlamentarias, excepto para el caso argentino en que se han tomado las de 2009 y en el caso nicaragüense las de 2006. Con esta acotación temporal se hace coincidir lo más posible la fecha de obtención de los datos de opinión pública de la confianza en los $\mathrm{OE}$, con los que se ha calculado la variable dependiente (encuesta de LAPOP de 2010), y la fecha de medición de la competitividad electoral.

Polarización ponderada parlamentaria. La distancia ideológica entre los dos partidos con posiciones más extremas en el Parlamento de cada país tiene efectos sobre la valoración de las instituciones (Sartori, 1980). En contextos muy polarizados se suele instalar un juego de desconfianzas mutuas entre las élites que afecta a las percepciones de los ciudadanos. Es probable que estas tendencias centrífugas aumenten la desconfianza respecto de los $\mathrm{OE}$ por ser percibidos como leales a una de las partes o polos enfrentados (Brenes, 2011). Igual que en el caso anterior, los datos de polarización han sido tomados de OPAL sobre el período electoral más cercano a 2010. 
Volatilidad electoral. La existencia de apoyos estables a los partidos políticos por parte del electorado contribuye a generar estabilidad en el sistema político (Mainwaring y Zoco, 2007). De ahí que la baja volatilidad tenga un efecto positivo sobre la confianza en los OE. Otra de las lecturas sobre los efectos de la volatilidad es la que realizan Hartlyn, McCoy y Mustillo (2009), que consideran la baja volatilidad como indicador de fortaleza de la organización partidista, hecho que puede ayudar a impedir el fraude electoral facilitando la actuación de los OE. En ambos casos la dirección esperada de variación es que a menores niveles de volatilidad más confianza en los OE. Los datos proceden también de OPAL y corresponden al período electoral más cercano a 2010.

Los valores concretos que adquieren las variables político-electorales en todos los casos se recogen en la Tabla 3 .

\section{La cultura política}

La valoración que los ciudadanos latinoamericanos realizan de las diferentes instituciones políticas está influida por la cultura política de cada país (Corral, 2008). De este modo, la confianza que específicamente generan los OE entraría dentro de esta relación de causalidad. Para medir el efecto de la cultura política sobre los niveles de confianza

Tabla 3. Variables político-electorales

\begin{tabular}{lccc}
\hline & $\begin{array}{c}\text { Competitividad } \\
\text { electoral }\end{array}$ & $\begin{array}{c}\text { Polarización parlamentaria } \\
\text { ponderada }\end{array}$ & $\begin{array}{c}\text { Volatilidad } \\
\text { electoral }\end{array}$ \\
\hline Argentina & 0,5 & 0,44 & 16,25 \\
Bolivia & 37,76 & 3,91 & 52,02 \\
Brasil & 3,9 & 1,88 & 15,61 \\
Chile & 5,22 & 3,09 & 11,85 \\
Colombia & 4,43 & 4,18 & 38,36 \\
Costa Rica & 19,7 & 0,13 & 22,86 \\
Ecuador & 31 & 4,29 & 36,42 \\
El Salvador & 3,4 & 11,70 & 15,15 \\
Guatemala & 6,3 & 1,44 & 41,16 \\
Honduras & 18,6 & 1,88 & 7,26 \\
México & 8 & 3,14 & 23,67 \\
Nicaragua & 6,6 & 6,01 & 12,96 \\
Panamá & 12,3 & 1,47 & 26,19 \\
Paraguay & 2 & 0,13 & 29,74 \\
Perú & 0,67 & 1,56 & 45,78 \\
R. Dominicana & 3,24 & 0,03 & 21,53 \\
Uruguay & 19,4 & 2,06 & 19,93 \\
Venezuela & 1,03 & 1,17 & 41,30 \\
\hline
\end{tabular}

Fuente: elaboración propia. Datos electorales para cálculo de competitividad, polarización y volatilidad obtenidos de OPAL y de Alcántara y Tagina (2011). 
en los OE se utilizan dos variables: la experiencia democrática y la confianza en las instituciones.

Experiencia democrática. Los años que un sistema político ha funcionado de forma democrática condicionan la valoración de las instituciones que conforman su régimen democrático. Cuanto mayor es la trayectoria democrática de un país más probabilidad hay de que se genere algún grado de consenso favorable al funcionamiento de sus instituciones, incluidos los OE. Ello se debe al aprendizaje del ciudadano respecto del valor y aportaciones de estas instituciones a la estabilidad democrática. La experiencia democrática se mide a partir del recuento de años de democracia realizado por Smith (2000), tomando como límite de la edad democrática de cada país el año 2000.

Confianza en las instituciones. La confianza de los ciudadanos en el sistema tiene efectos sobre la confianza en las elecciones (Layton, 2010). Este argumento se podría trasladar a la confianza específica en los OE. Así, sería esperable que la confianza de los ciudadanos en otras instituciones relevantes del sistema afecte a la valoración específica de los OE. De modo que los contextos de desconfianza generalizada serán poco proclives a generar valoraciones positivas de los OE electorales, y viceversa. Es verdad que se podría hablar de cierto nivel de endogeneidad por arrastre, ya que son todas valoraciones de instituciones. Aún así, es defendible que la existencia de un entorno de confianza general en las principales instituciones del país, medida mediante la confianza en instituciones no electorales, tenga efectos sobre la generación de confianza en una institución específica. Se recurre a datos de confianza en el Parlamento y en el sistema judicial, de LAPOP 2010, para obtener una medición agregada de la confianza en las instituciones.

La Tabla 4 muestra los valores de cada uno de los casos en las variables de cultura política que se acaban de presentar.

\section{Tabla de verdad}

La tabla de verdad construida con estos datos permite valorar el impacto de las variables de tipo cualitativo, relacionadas con el diseño institucional, junto con las de tipo cuantitativo, referidas a la dinámica político-electoral y a la cultura política (Tabla 5).

La hipótesis que sostenemos es que para el logro de OE en los que los ciudadanos confíen, la existencia de un diseño institucional que permita autonomía del OE respecto de otros poderes y actores en el ejercicio de sus funciones y decisiones es más relevante que la especialización de funciones entre distintos órganos electorales. Este segundo factor ha tendido a sobreestimarse en las reformas recientes acontecidas en varios $\mathrm{OE}$ (Ecuador y República Dominicana, entre otros). Asimismo, esperamos encontrar que en contextos político-electorales conflictivos, así como en entornos con baja confianza en las instituciones, aumente la desconfianza hacia los OE.

Igual que las variables de diseño institucional que ya se habían dicotomizado anteriormente, se realiza la codificación binaria del resto de variables independientes con valores de 0 (ausencia de la propiedad) y 1 (existencia de la propiedad). Así, se ha asignado un valor de 0 a la baja competitividad (más de siete puntos de diferencia entre el primer 
Tabla 4. Variables relacionadas con la cultura política

\begin{tabular}{lcccc}
\hline & $\begin{array}{c}\text { Años de democracia } \\
\text { (hasta 2000) }\end{array}$ & $\begin{array}{c}\text { Confianza en } \\
\text { el Parlamento }\end{array}$ & $\begin{array}{c}\text { Confianza en } \\
\text { sistema judicial }\end{array}$ & $\begin{array}{c}\text { Agregado de confianza } \\
\text { en instituciones }\end{array}$ \\
\hline Argentina & 40 & 37,4 & 36,9 & 37,15 \\
Bolivia & 26 & 47,2 & 43,5 & 45,35 \\
Brasil & 27 & 35,8 & 45,2 & 40,5 \\
Chile & 52 & 50,5 & 45,8 & 48,15 \\
Colombia & 50 & 51,6 & 56,3 & 53,95 \\
Costa Rica & 48 & 47,6 & 52,0 & 49,8 \\
Ecuador & 30 & 21,8 & 35,5 & 28,65 \\
El Salvador & 7 & 40,3 & 45,9 & 43,1 \\
Guatemala & 14 & 40,2 & 44,9 & 42,55 \\
Honduras & 3 & 41,1 & 41,3 & 41,2 \\
México & 3 & 55,6 & 50,8 & 53,2 \\
Nicaragua & 11 & 36,5 & 41,4 & 38,95 \\
Panamá & 7 & 38,0 & 43,2 & 40,6 \\
Paraguay & 8 & 24,5 & 23,3 & 23,9 \\
Perú & 25 & 33,2 & 33,9 & 33,55 \\
R. Dominicana & 22 & 53,7 & 50,1 & 51,9 \\
Uruguay & 65 & 53,6 & 54,9 & 54,25 \\
Venezuela & 44 & $*$ & $*$ & $*$ \\
\hline
\end{tabular}

* datos no disponibles

Fuente: elaboración propia. Datos sobre edad de la democracia de Smith (2000); datos confianza de LAPOP 2010.

y el segundo partido más votado), a la baja polarización (menos de tres puntos de distancia entre los dos partidos con posiciones más extremas), a la baja-media volatilidad (menos de veinte puntos), a los casos donde la experiencia democrática no ha sido larga (menos de quince años de trayectoria democrática previa al año 2000) y a la ausencia de confianza en las instituciones (menos de $40 \%$ de confianza). Del mismo, se ha dado un valor de 1 a las situaciones inversas (alta competitividad, alta polarización, alta volatilidad, media-alta experiencia democrática y media-alta confianza). A su vez, la variable dependiente está clasificada en tres valores: alta, media y baja confianza en los organismos electorales, según los datos de LAPOP de 2010. Se ha preferido no forzar un planteamiento dicotómico para los valores de la variable de confianza en los $\mathrm{OE}$ y optar por una clasificación más fiel al modo en que los países se distribuyen en la respuesta.

\section{2. Resultados del análisis: ¿hay patrones explicativos?}

Los resultados de la tabla de verdad han sido traducidos a ecuaciones donde se asigna a cada rasgo una letra: mayúscula si existe ese rasgo y minúscula si está ausente (Tabla 6). Por ejemplo, "A" denota especialización de funciones y "a" ausencia de especialización. 


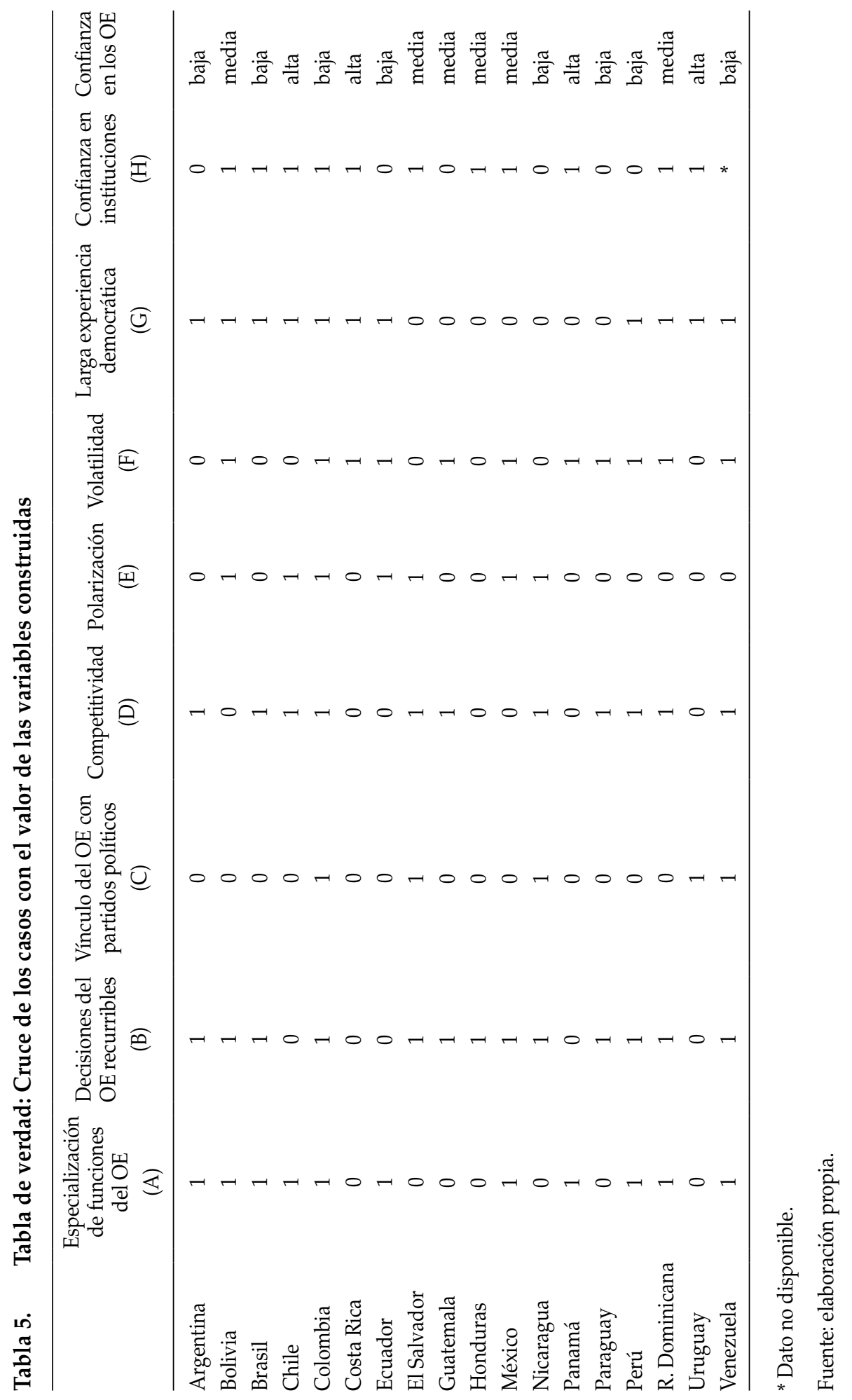


La tabla de verdad hace posible ver la coincidencia entre combinaciones de valores de las variables independientes y el valor que adquiere la dependiente a lo largo de los casos. Aunque se trate de un método exploratorio, con esta información nos aproximamos al grado y dirección de la influencia de las variables independientes sobre la variable dependiente.

Las Tablas 5 y 6 muestran que los países con niveles elevados de confianza en los $\mathrm{OE}$ comparten dos características: no hay posibilidad de recurrir las decisiones de los $\mathrm{OE}$, por lo que el $\mathrm{OE}$ es autónomo respecto de otros poderes, y hay un entorno de alta confianza institucional. Por lo tanto, un rasgo del diseño institucional combinado con un rasgo de la cultura política del entorno tendrían responsabilidad en la generación de OE confiables para los ciudadanos.

Por su parte, los OE con bajos niveles de confianza comparten tanto el rasgo de que sus decisiones pueden ser recurridas en otras instancias (excepto en el caso de Ecuador), como una competitividad electoral con resultados muy ajustados entre los partidos

Tabla 6: Ecuaciones de las variables independientes y valor de la variable dependiente

\begin{tabular}{llc}
\hline Ecuación & $\begin{array}{c}\text { Valor de la variable dependiente: } \\
\text { confianza en los OE }\end{array}$ \\
\hline Argentina & ABcDefGh & baja \\
Paraguay & aBcDeFgh & baja \\
Nicaragua & ABCDEfgh & baja \\
Ecuador & AbcdEFGh & baja \\
Perú & ABcDeFGh & baja \\
Venezuela & ABCDeFG* & baja \\
Colombia & ABCDEFGH & baja \\
Brasil & ABcDefGH & baja \\
Guatemala & aBcDeFgh & media \\
El Salvador & aBCDEfgH & media \\
Honduras & aBcdefgH & media \\
México & ABcdEFgH & media \\
Bolivia & ABcdEFGH & media \\
R. Dominicana & ABcDeFGH & media \\
Chile & AbcDEfGH & alta \\
Panamá & AbcdeFgH & alta \\
Costa Rica & abcdeFGH & alta \\
Uruguay & abCdefGH & alta \\
\hline
\end{tabular}

* Dato no disponible.

Fuente: elaboración propia. 
políticos (excepto Ecuador que no tiene alta competitividad electoral, aunque tendría una dinámica conflictiva como muestra su alta polarización). Nuevamente, la autonomía que permite el diseño institucional aparece como variable relevante, junto con un rasgo del entorno político-electoral.

La excepcionalidad de Ecuador en los efectos de la variable del diseño institucional puede ser explicada porque los cambios de la Constitución de 2008 que afectaban a la organización electoral (entre otras cosas, se llevó a cabo una desconcentración de las funciones, la despartidización de los miembros de los OE y la eliminación de la revisión de las decisiones en materia electoral) son aún recientes para elevar los niveles de confianza de los ciudadanos (medida en 2010). Además, Ecuador no tiene un entorno de alta confianza en las instituciones, a diferencia de los casos con alta confianza en los OE.

Del análisis anterior y atendiendo al caso excepcional de Ecuador, se extrae una conclusión: la variable de confianza institucional constituye un factor necesario, aunque insuficiente de forma aislada. ${ }^{8}$ Esto significa que todos los casos de alta confianza en los OE tienen un entorno de alta confianza institucional, pero que la existencia de esta no asegura la existencia de OE con alto grado de confianza.

Los hallazgos respecto de la relevancia de la revisión de las decisiones, de la competitividad y del entorno de confianza institucional se aprecian de forma más clara si se centra la atención en los tres casos con más desconfianza y menos desconfianza en los OE. Así, en Argentina, Paraguay y Nicaragua las decisiones de los OE son revisables, hay alta competitividad y baja confianza institucional; mientras que Uruguay, Panamá y Costa Rica tienen OE con decisiones no revisables por otros poderes, se da baja competitividad electoral y están insertos en entornos de alta confianza institucional. Además, los tres OE concentran las funciones administrativas y contenciosas en una única institución, como Thompson y Valverde (2007) ya hicieron notar. Esta falta de especialización de algunos de los OE que gozan de alta confianza de los ciudadanos contradice las expectativas que han guiado el rumbo de las reformas en algunos países y confirma el argumento de Mozzafar y Schedler (2002) sobre los efectos inciertos de la especialización de las funciones. En este sentido, dicho factor no consigue de forma aislada mejorar la confianza en los $\mathrm{OE}$, sino que necesita, como aquí defendemos, de una cultura política de confianza institucional y un entorno político-electoral que no sea muy conflictivo.

Finalmente, los países con niveles intermedios de confianza no tienen un patrón explicativo claro, salvo que las decisiones de sus OE pueden ser recurridas. En las demás variables presentan una notable variación, incluido en los niveles de competitividad electoral que era una variable que identificaba al grupo de casos con baja confianza en los OE, así como en la confianza institucional que era una variable común para el grupo de casos de alta confianza en los OE.

$8 \quad$ Respecto de las condiciones de necesidad y suficiencia, en palabras de Pérez Liñán (2010: 139-140): “La regla para identificar necesidad es simple: si X es condición necesaria para $Y$, todas las instancias de $Y$ deben mostrar la presencia de $X(\ldots)$ La regla para identificar suficiencia invierte así el criterio de necesidad: si la configuración causal $\mathrm{X}$ es condición suficiente para $Y$, todas las instancias de $X$ deben ofrecer también la presencia de $Y^{\prime \prime}$. 
Cabe señalar que los hallazgos relativos a todas estas variables van en la dirección de lo esperado por la teoría, excepto el de la especialización de funciones donde la propia teoría no tiene expectativas claras. Por otra parte, como muestra la tabla de verdad, ninguna característica de las analizadas es una condición necesaria y suficiente de forma aislada. No obstante, un entorno con confianza institucional parece tener efectos decisivos combinado con la imposibilidad de recurrir a decisiones de los OE y la existencia de baja competitividad electoral, aunque esto último tenga una menor capacidad predictiva.

La utilidad del análisis booleano ha residido en ver las combinaciones de factores que pueden llevar a mayores o menores niveles de confianza en los OE. Estas tendencias pueden ser contrastadas en el futuro con análisis confirmatorios como el análisis de regresión. Para ello, sería necesario incluir un mayor número de casos mediante mediciones de las variables en diferentes momentos en el tiempo para cada país. Asimismo, para la agenda futura de investigación queda pendiente el análisis de los efectos de los cambios en el diseño institucional o en las variables político-electorales sobre los niveles de confianza ciudadana en los OE.

\section{EL IMPACTO DE LA CONFIANZA EN LOS ORGANISMOS ELECTORALES SOBRE LA CALIDAD DE LA DEMOCRACIA}

En la última década, un nuevo tema ha emergido con fuerza en el campo de la política comparada latinoamericana: la calidad democrática. Varios estudios se han ocupado de medir la calidad de las democracias de la región y determinar las causas de los diferentes niveles de calidad observados (Altman y Pérez-Liñán, 2002; Mainwaring y Pérez-Liñán, 2008; Levine y Molina, 2011; Barreda, 2011). En este escenario situamos el análisis de los posibles efectos políticos de la confianza en los OE. Se pretende examinar si la confianza en estos organismos tiene algún impacto significativo en la calidad de las democracias latinoamericanas. De acuerdo con los argumentos y evidencias de la literatura sobre confianza institucional señalados, cabe esperar un impacto positivo de la confianza en los OE sobre la calidad democrática. No obstante, conviene advertir que la percepción sobre los OE puede estar influenciada, en parte, por la percepción del nivel de calidad democrática del país. Hay riesgo, pues, de que haya una relación de endogeneidad entre estas dos cuestiones institucionales, tal como se ha constatado en diversos estudios. ${ }^{9}$ Para paliar esta situación se adoptarán algunas medidas metodológicas, como luego se comentará.

La variable independiente del análisis es la confianza ciudadana en los OE, medida con el indicador de LAPOP. La delimitación de la variable dependiente resulta mucho más complejo, debido a la diversidad semántica y la multidimensionalidad del concepto de calidad democrática (Diamond y Morlino, 2004; Levine y Molina, 2011).

9 Por ejemplo, Birch (2008) encuentra -a diferencia de otros estudios- que la independencia de los OE tiene un efecto negativo sobre la credibilidad de las elecciones. La razón es que en algunos países se reformó el diseño de los OE como respuesta a problemas percibidos de imparcialidad en las elecciones. 
Son muchos los indicadores susceptibles de medir la calidad de una democracia. A la hora de seleccionarlos se han tenido en cuenta dos criterios. El primero es que se trate de indicadores reconocidos en la literatura y que reflejen la diversidad de concepciones de calidad democrática. Dentro de esta diversidad se pueden identificar dos grupos de definiciones: aquellas inspiradas en la noción de poliarquía de Dahl (2002) y que conciben la democracia como un conjunto de procedimientos que regulan el acceso al poder político (Altman y Pérez-Liñán, 2002); y las que plantean que la democracia va más allá del acceso al poder político y que es necesario atender también al ejercicio del poder político, particularmente a los mecanismos de control político (O'Donnell, 2001). El segundo criterio de selección es que no se trate de indicadores esporádicos, sino que tengan una cierta continuidad temporal.

De acuerdo con lo anterior, se han seleccionado cinco indicadores utilizados en la literatura sobre calidad democrática (Levine y Molina, 2011; Barreda, 2011). Dos están relacionados con los parámetros básicos de una poliarquía: el indicador de derechos políticos de FH, para medir la garantía de derechos democráticos; y el de satisfacción con el funcionamiento de la democracia de Latinobarómetro, para medir el nivel de atención a preferencias ciudadanas (responsiveness). Un tercer indicador es el índice de democracia institucionalizada de Polity IV, que no solo mide cuestiones relativas al acceso al poder político, sino también cuestiones relativas a su desempeño, como la existencia de constricciones al ejercicio del poder ejecutivo (imperio de la ley, control por parte del legislativo, etc.). Otro indicador es el índice de percepción de corrupción de Transparencia Internacional, que ofrece información sobre la eficacia de los mecanismos de control del poder político. Finalmente, se ha seleccionado el índice de calidad democrática de Barreda (2011), resultado de agregar indicadores correspondientes a garantía de derechos democráticos, responsiveness, participación, Estado de derecho y accountability.

En el análisis estadístico se han introducido tres variables de control: el nivel educativo, la fragmentación étnica y la experiencia democrática. Del nivel educativo se ha destacado su influencia positiva en la calidad democrática, entre otras razones, porque incide significativamente en la capacidad de tomar una decisión informada (Levine y Molina, 2011). Diversos trabajos han señalado que la elevada fragmentación étnica socava la calidad democrática. En particular, se ha asociado la elevada fragmentación étnica con un mayor nivel de favoritismo político y restricción de la libertad de oposición (La Porta et al., 1999). Por último, otros estudios han resaltado el impacto positivo de la experiencia democrática, en la medida en que esta favorece el desarrollo de elementos institucionales y culturales democráticos (Mainwaring y Pérez-Liñán, 2008). Para medir estas tres variables se han seleccionado los indicadores de alfabetismo (UNESCO), fragmentación étnica (Alesina et al., 2002) y años de democracia (Smith, 2004). En los dos últimos casos se dispone de información para un año, pero se trata de indicadores de carácter estructural, por lo que se asumirán como constantes durante el periodo examinado.

El conjunto de países es muy pequeño para realizar un análisis estadístico mínimamente fiable. Por ello, se consideran los valores de los indicadores de cada país en diferentes años. En concreto, se toma como referencia la serie temporal de la confianza en $\mathrm{OE}$, lo que permite ampliar el tamaño de la $\mathrm{N}$ hasta 72 (18 países por 4 años). Los indicadores 
de la variable dependiente constituyen un caso particular. Con la intención de precisar las relaciones de causalidad y paliar los problemas de endogeneidad mencionados, se tratan las variables independientes como variables rezagadas respecto de la variable dependiente (con una referencia temporal previa a la dependiente). Así, por ejemplo, se asocia el valor de la confianza en OE en 2008 con su posible efecto sobre la corrupción en el periodo siguiente (2010). Al aplicar este criterio queda reducida la serie temporal de los indicadores de la variable dependiente, ya que no se dispone de suficiente información posterior a 2010.

En la Tabla 7 se presentan los resultados del análisis de regresión lineal realizado. Debido al número reducido de observaciones y el riesgo de que haya relaciones de endogeneidad (disminuido por el uso de variables rezagadas) conviene alertar sobre las limitaciones del análisis y subrayar su carácter exploratorio. Como muestran los coeficientes tipificados de los cinco modelos que utilizan indicadores alternativos de calidad democrática, el impacto de la confianza en los $\mathrm{OE}$ es estadísticamente significativo en todos los casos. En especial, destaca la mayor capacidad explicativa respecto del índice de calidad democrática. El signo del coeficiente de confianza ciudadana es en todos los casos positivo, como se esperaba. Por tanto, el análisis pone de manifiesto una contribución

Tabla 7. Determinantes de 5 indicadores de calidad democrática (análisis de regresión lineal)

\begin{tabular}{lccccc}
\hline \multirow{2}{*}{$\begin{array}{c}\text { Variables } \\
\text { independientes }\end{array}$} & $\begin{array}{c}\text { Derechos } \\
\text { políticos }\end{array}$ & $\begin{array}{c}\text { Satisfacción } \\
\text { democracia }\end{array}$ & $\begin{array}{c}\text { Democracia } \\
\text { institucionalizada }\end{array}$ & $\begin{array}{c}\text { Percepción } \\
\text { corrupción }\end{array}$ & $\begin{array}{c}\text { Índice calidad } \\
\text { democrática }\end{array}$ \\
\cline { 2 - 6 } Confianza en OE & $0,451^{* * *}$ & $0,278^{* *}$ & $0,388^{* * *}$ & $0,455^{* * *}$ & $0,501^{* * * *}$ \\
& $(4,367)$ & $(2,507)$ & $(2,853)$ & $(5,180)$ & $(4,277)$ \\
Alfabetismo & $0,471^{* * *}$ & 0,069 & $-0,031$ & $-0,001$ & $0,276^{*}$ \\
Fragmentación & $(3,848)$ & $(0,522)$ & $(-0,195)$ & $(-0,013)$ & $(1,965)$ \\
étnica & $-0,276^{* * *}$ & $-0,212^{* *}$ & $-0,398^{* * *}$ & $-0,342^{* * *}$ & $-0,237^{* *}$ \\
Años de & $(-2,819)$ & $(-2,015)$ & $(-3,020)$ & $(-4,104)$ & $(-2,095)$ \\
democracia & $-0,104$ & $0,428^{* * *}$ & $-0,139$ & $0,399^{* * *}$ & 0,154 \\
Constante & $(-0,809)$ & $(3,109)$ & $(-0,847)$ & $(3,659)$ & $(1,058)$ \\
N ${ }^{o}$ observaciones & $(-1,353)$ & $(0,105)$ & $(1,820)$ & $(-0,134)$ & $(-3,447)$ \\
$R^{2}$ corregida & 54 & 54 & 51 & 54 & 36 \\
\hline
\end{tabular}

${ }^{*},{ }^{* *} \mathrm{y}^{* * *}$ indican niveles de significación del 90,95 y $99 \%$, respectivamente. Los valores corresponden a coeficientes Beta y los que están entre paréntesis a coeficientes t. Se ha invertido la escala original de derechos políticos (ahora 7 es el máximo y 1 el mínimo). En el caso de la percepción de corrupción 10 indica "altamente transparente" y 0 "altamente corrupto". En todas las variables dependientes los valores van en la misma dirección: cuanto más altos mejores resultados en calidad democrática.

Fuente: elaboración propia. 
positiva de la confianza en los OE sobre la calidad de las democracias latinoamericanas. Conviene anotar también que la influencia de las variables de control se ajusta a lo esperado: la fragmentación étnica -la más explicativa- tiene un efecto negativo en la calidad democrática, justo lo contrario de lo que sucede con el nivel educativo y los años de democracia. En un análisis preliminar se tuvo en cuenta la incidencia de la dimensión temporal mediante variables dummy, pero estas no resultaron significativas. En otras palabras, el factor "tiempo" no influye de manera significativa en la variación de los aspectos de calidad democrática examinados.

\section{CONCLUSIONES}

En los últimos años, el estudio de los OE despierta un creciente interés en la ciencia política, pero todavía hay una insuficiencia notable de análisis empíricos en esta materia. De las diferentes avenidas para la investigación de los OE nos hemos centrado en la confianza en los $\mathrm{OE}$, explorando algunos de los factores que tienen incidencia sobre ella, así como el impacto de la confianza en los OE sobre la calidad de la democracia. Nos hemos ocupado, por lo tanto, de la cadena causal completa relativa a la confianza en los OE. Para ello se ha optado por una combinación de datos y técnicas cualitativas (análisis booleano) y cuantitativas (análisis descriptivo y de regresión) que es novedosa en la literatura. Los principales hallazgos del estudio realizado son tres.

En primer lugar, la confianza en los $\mathrm{OE}$ latinoamericanos ofrece un panorama alentador. Los OE figuran entre las instituciones mejor valoradas por parte de la ciudadanía de América Latina y esta valoración prácticamente no ha variado entre 2004 y 2010. Esta percepción positiva de los OE también es compartida por los diputados encuestados. Donde sí se aprecian notables variaciones es al establecer una comparación entre países. En concreto, se pueden identificar tres grandes grupos: Chile, Panamá, Costa Rica y, sobre todo, Uruguay, con los niveles más elevados de confianza ciudadana; Argentina, Paraguay, Nicaragua, Ecuador, Perú, Venezuela, Colombia y Brasil, con los niveles más bajos de confianza, especialmente los tres primeros; y los demás países situados en una zona intermedia.

La segunda contribución hace referencia a los factores que explican la variación en los niveles de confianza en los OE de unos países a otros. La literatura ha puesto de manifiesto causas muy diversas, pero pocos trabajos las han examinado empíricamente y menos aún de una forma integral. En este estudio hemos analizado la incidencia de un conjunto diverso de factores, que conciernen a tres aspectos: el diseño institucional de los OE, el contexto político-electoral en que operan y la cultura política del país. El análisis muestra que ninguno de los factores examinados constituye por sí solo una causa necesaria y suficiente del nivel de confianza ciudadana en los OE. Esto último es resultado de una combinación de causas que varían de unos países a otros. No obstante, se ha constatado la influencia significativa de tres factores: el margen de autonomía de los OE en el ejercicio de sus funciones, el nivel de confianza en las principales instituciones del país y el nivel de competitividad electoral (esto último en la mayor parte de los casos 
de menor confianza en los $\mathrm{OE}$ ). En los tres casos la dirección explicativa se ajusta a lo esperado. Así, el nivel de independencia de los $\mathrm{OE}$ contribuye a un mejor rendimiento de los mismos y, consecuentemente, a un nivel de confianza ciudadano más favorable. En cambio, un entorno de alta competitividad electoral y de desconfianza institucional suele repercutir desfavorablemente en la confianza en los OE.

En tercer lugar, y teniendo presente las limitaciones del análisis estadístico realizado, el estudio ha mostrado la relación existente entre la confianza ciudadana en los OE y la calidad de la democracia. Se trata de una conclusión que no es menor, ya que aunque forma parte del saber convencional, no ha sido suficientemente comprobada de forma empírica. Nuestro análisis evidencia que el nivel de confianza ciudadana en los OE ejerce un impacto positivo sobre la calidad de una democracia, tanto si se consideran parámetros de calidad minimalistas (circunscritos a la cuestión del acceso al poder político) como parámetros más amplios (relativos al ejercicio del poder político).

Con estas conclusiones se puede afirmar que el trabajo en la mejora del diseño y procedimientos de actuación de los OE que de forma sostenida se lleva a cabo en la región, repercutirá en la confianza en los $\mathrm{OE}$ y, por ende, en el aumento de la calidad de la democracia. Sin embargo, no se debe pensar que todo es resultado de la ingeniería institucional. Como se ha visto, el entorno político-electoral y la cultura política de cada país son elementos que inciden en el nivel de confianza ciudadana en los OE. En definitiva, cualquier estudioso o policy maker de los OE latinoamericanos no solo debe centrar su atención en el diseño y funcionamiento de los $\mathrm{OE}$, sino también en el entorno institucional en que estos organismos operan.

\section{REFERENCIAS}

Alcántara y Tagina (eds.). 2011. Política y elecciones en América Latina en tiempos de los Bicentenarios (20092010). Madrid: Centro de Estudios Políticos y Constitucionales.

Alesina, Alberto et al. 2003. "Fractionalization". Journal of Economic Growth 8 (2): 155-194.

Altman, David y Aníbal Pérez-Liñán. 2002. "Assessing the Quality of Democracy: Freedom, Competitiveness and Participation in Eighteen Latin American Countries". Democratization 9 (2): 85-100.

Araujo, Walter, et al. 2008. "Resultados electorales ajustados. Experiencias y lecciones aprendidas". Cuaderno de CAPEL 52.

Aydin, Aylin y Cerem I. Cenker. 2012. "Public Confidence in Government: Empirical Implications from a Developing Democracy". International Political Science Review 33 (2): 230-250.

Barreda, Mikel. 2011. "La calidad de la democracia: Un análisis comparado de América Latina". Política y Gobierno 18 (2): 265-295.

Barrientos, Fernando. 2010. “Confianza en las Elecciones y el Rol de los Organismos Electorales en América Latina". Revista de Derecho Electoral 10: 1-35.

Barrientos, Fernando. 2011. Gestión Electoral Comparada y Confianza en las Elecciones en América Latina. México, DF: Instituto Nacional de Administración Pública.

Beltrán, Ulises. 2009. “Introducción. El proceso electoral: Precampañas, campañas y resultado”. Política y Gobierno vol. temático: 5-39.

Birch, Sarah. 2008. “Electoral Institutions and Popular Confidence in Electoral Processes: A Cross-national Analysis". Electoral Studies 27 (2): 305-320. 
Brenes, Diego. 2009. "(Des)confianza en los organismos y procesos electorales en América Latina". Boletín datos de opinión, Proyecto Élites Parlamentarias Latinoamericanas, Universidad de Salamanca.

Brenes, Diego. 2011. "El rol político del juez electoral. El Tribunal Supremo de Elecciones de la República de Costa Rica. Un análisis comparado". Tesis Doctoral, Universidad de Salamanca.

Corral, Margarita. 2008. "Desconfianza en los partidos políticos en América Latina", Revista de Ciencia Política 28 (2): 195-202.

Dahl, Robert. 2002. La poliarquía: participación y oposición. Madrid: Tecnos.

Diamond, Larry y Leonardo Morlino. 2004. "The Quality of Democracy. An Overview". Journal of Democracy 15 (4): 20-31.

Easton, David. 1975. "A Re-Assessment of the Concept of Political Support". British Journal of Political Science 5 (4): 435-457.

Hartlyn, Jonathan. 1998. The Struggle for Democratic Politics in the Dominican Republic. Chapel Hill: University of North Carolina Press.

Hartlyn, Jonathan, Jenniffer McCoy y Thomas M. Mustillo. 2009. “La importancia de la gobernanza electoral y la calidad de las elecciones en la América Latina contemporánea". América Latina Hoy (51): 15-40.

La Porta, Rafael, et al. 1999. "The Quality of Government". The Journal of Law, Economics \& Organization 15 (1): 222-279.

Lehoucq, Fabrice E. e Iván Molina. 2002. Stuffing the Ballot Box: Fraud, Electoral Reform and Democratization in Costa Rica. Nueva York: Cambridge University Press.

Levine, Daniel H. y José E. Molina. 2011. "Evaluating the Quality of Democracy in Latin America" y "Measuring the quality of democracy". En The Quality of Democracy in Latin America, editado por Daniel H. Levine y José E. Molina, 1-37. Boulder: Lynne Rienner Publishers.

Layton Matthew L. 2010. "Trust in Elections". Americas Barometer Insights 37. Vanderbilt University.

López Pintor, Rafael. 2000. Electoral Management Bodies as Institutions of Governance. Nueva York: PNUD.

Mainwaring, Scott y Edurne Zoco. 2007. "Secuencias políticas y estabilización de la competencia partidista: volatilidad electoral en viejas y nuevas democracias". América Latina Hoy 46: 147-171.

Mainwaring, Scott y Aníbal Pérez-Liñán. 2008. "Regime Legacies and Democratization: Explaining Variance in the Level of Democracy in Latin America, 1978-2004". Working Paper del Kellogg Institute for International Studies 354.

Molina, José y Janeth Hernández. 1998. "La credibilidad de las elecciones latinoamericanas y sus factores. El efecto de los OE, el sistema de partidos y las actitudes políticas". Paper presentado en XXI Congreso de LASA, Chicago.

Moreno, Erika, Grian F. Crisp y Matthew S. Shugart. 2003. "The accountability deficit in Latin America". En Democratic Accountability in Latin America, editado por Scott Mainwaring y Christopher Welna, 79-131. Nueva York: Oxford University Presss.

Mozaffar, Shaheen y Andreas Schedler. 2002. "The Comparative Study of Electoral Governance-Introduction". International Political Science Review 23 (1): 85-101.

Newton, Kenneth y Pippa Norris. 2000. "Confidence in Public Institutions: Faith, Culture or Performance?". En Disaffected Democracies. What's Troubling the Trilateral Countries?, editado por Susan J. Pharr y Robert D. Putnam, 52-73. Princeton: Princeton University Press.

O'Donnell, Guillermo. 2001. "La irrenunciabilidad del Estado de Derecho". Instituciones y Desarrollo (8-9): 43-82.

Orozco, J. Jesús. s. f. "El contencioso electoral en América Latina", IIDH. Edición Costa Rica. Disponible en: http:/ /www.iidh.ed.cr/comunidades/redelectoral/docs/red_publinea/Tratado/ Contencioso\%20Electoral.htm

Pérez Liñán, Aníbal (2010): "El método comparativo y el análisis de configuraciones causales". Revista Latinoamericana de Política Comparada 3, enero: 15-43.

Picado, Hugo. 2006. “Calidad de las elecciones en Latinoamérica: la independencia formal de los Tribunales Electorales en México, Centroamérica y Panamá", mimeo.

Putnam, Robert, Susan J. Pharr y Russell J. Dalton. 2000. "Introduction: What's Troubling the Trilateral Democracies?". En Disaffected Democracies. What's Troubling the Trilateral Countries?, editado por Susan J. Pharr y Robert D. Putnam, 3-27. Princeton: Princeton University Press. 
Ragin, Charles C. 1987. The Comparative Method. Moving beyond qualitative and quantitative strategies. Berkeley: University of California Press

Sartori, Giovanni. 1980. Partidos y sistemas de partidos. Madrid: Alianza editorial.

Sobrado, Luis A. 2008. "Los organismos electorales: autonomía, formación electoral y democracia”. Revista de Derecho Electoral 6: 1-20.

Sonnleitner, Willibald. 2007. "Élections serrées dans des contextes polarisés". En Amérique latine, les élections contre la démocratie?, editado por Olivier Dabène, 131-160. Paris: Editorial Presses de Sciences Po.

Smith, Peter H. 2004. “Los ciclos de democracia electoral en América Latina, 1900-2000”. Política y Gobierno 11 (2): 189-228.

Struwig, Jare, Benjamin Roberts y Elme Vivier. 2011. "A Vote of Confidence: Election Management and Public Perceptions of Electoral Processes in South Africa". Journal of Public Administration 46 (3.1): 1122-1138.

Thompson, José y Ricardo Valverde. 2007. “Organización electoral en América Latina. Avances, retos y perspectivas", Taller organizado por Transparencia Internacional, Lima. http:/ /www.transparencia. org.pe/documentos/organizacion_electoral_en_america_latina._j_thompsom.pdf

Wall, Alan et al. 2006. Electoral Management Design: The International IDEA Handbook. Estocolmo: International IDEA.

Weil, Frederick D. 1989. “The Sources and Structure of Legitimation in Western Democracies: A Consolidated Model Tested with Time-series Data in Six Countries since World War II". American Sociological Review 54 (5): 682-706.

Mikel Barreda es profesor agregado de Ciencia Política de la Universitat Oberta de Catalunya (Barcelona). Doctor en Ciencias Políticas y Sociología por la Universidad de Deusto (Bilbao). Entre sus publicaciones recientes figuran la edición del libro Las instituciones políticas de las democracias latinoamericanas (editoriales Plural y Huygens, en prensa), la coedición de Gobernanza, Instituciones y Desarrollo (Tirant lo Blanch, 2012) y los artículos "La calidad de la democracia en América Latina: un análisis comparado" (Política y Gobierno, 2011) y "La calidad de la democracia en Paraguay: un avance sobre caminos tortuosos" (América Latina Hoy, 2010). E-mail: mbarreda@uoc.edu

Leticia M. Ruiz Rodríguez es profesora titular de la Facultad de Ciencias Políticas y Sociología de la Universidad Complutense de Madrid. Doctora en Ciencia Política por la Universidad de Salamanca (USAL). Máster en Ciencia Política por la University of North Carolina en Chapel Hill, Máster en Estudios Latinoamericanos por la USAL y Diploma en Data Analysis and Collection por la University of Essex. Es autora del libro Partidos y coherencia. Parlamentarios en América Latina (CEPC, 2007) y coeditora de Chile. Política y modernización democrática (Edicions Bellaterra, 2006). Ha publicado artículos en Revista Española de Ciencia Política y Revista Española de Investigaciones Sociológicas, entre otras. E-Mail: leticiamaria.ruiz@cps.ucm.es 
